\title{
Regularities and Characteristics of Gel Fuel Ignition
}

\author{
Dmitrii Glushkov ${ }^{1}$, Pavel Strizhak ${ }^{1}$ \\ ${ }^{1}$ National Research Tomsk Polytechnic University \\ Lenina Avenue, 30, Tomsk, Russia, 634050 \\ dmitriyog@tpu.ru
}

\begin{abstract}
This experimental research studies the gas-phase ignition of single droplets of gel fuels based on ethyl alcohol with a gellant, liquid and fine solid combustible components. Droplets $2 \mathrm{~mm}$ in diameter were located on a holder and heated in a muffle furnace at a temperature ranging from 873 to $1073 \mathrm{~K}$. For fuel compositions under study, we determined the threshold conditions (minimum ambient temperature of 873-943 K) required for the gel fuel ignition as well as the dependences of the ignition delay times versus air temperature. The ignition delay times range from 0.1 to $3.3 \mathrm{~s}$. For the first time, using the shadow methods, we analyze the characteristics of vapor jetting during the induction period as a result of microexplosions caused by the differences in the boiling temperatures of fuel components. The average vapor jetting speed is about $3 \mathrm{~m} / \mathrm{s}$. The size of the zones, in which the vapors slow down to zero, ranges from 6 to 8 mm. The results obtained helped us formulate a physical model of the process, which may serve as the basis for the development of a mathematical model simulating the ignition of gel fuel droplets under rapid heating.
\end{abstract}

Keywords: Gel fuel; ignition delay time; vapour jetting; physical model.

\section{Introduction}

Solid and liquid fuels are widely used in practice nowadays. Each type has both advantages and disadvantages relative to one another. However, with the potential of solid and liquid fuels almost exhausted, gel fuels may become an alternative energy resource [1-4]. These fuels combine the advantages of the solids with those of liquids. The main advantages [1] of gel fuels in comparison to liquid and solid propellants are safety aspects (few vapors are released in case of leak or spill; low sensitivity to impact, friction and electrostatic discharge; low probability of accidental ignition, because combustion is controllable and can be prevented; cracks in the gel structure have no effect on increasing of burning area and uncontrolled combustion or explosion), performance aspects (relatively high specific impulse and density impulse; relatively simple energy management), and storage aspects (long term storage capability; flexible packing; relatively low particle sedimentation).

Depending on the environmental characteristics and the gel fuel destination, its state of the matter may vary significantly: from highly viscous liquid to solid. Within the combustion theory of solid and liquid condensed substances [5-8], the properties of composite solid propellants and liquid propellants have been fully explored. Mathematical models have also been developed to forecast the characteristics of physical and chemical transformations that they undergo when heated. Experimental research findings [1-4] reveal quite a significant difference in the physics and chemistry of the ignition and combustion of gel fuels as compared to typical solid and liquid propellants. Hence, researching gel fuels is a task of great current interest.

The purpose of this research is to study experimentally the heat-induced physical and chemical transformations in single droplets of gel fuels of various compositions based on ethyl alcohol with a gellant, liquid and fine solid combustible components.

\section{Materials}

Experimental studies were performed for four compositions of gel fuels based on a 95\% ethanol solution. Pine resin was added to liquid fuel as a gellant. We also performed some experiments without adding any components to compare and the ignition patterns of the gel fuel and its basic component. Table 1 shows the nomenclature for the initial fuel and different compositions of the gel fuel as well as its component ratio. 
Table 1: Component composition of fuels.

\begin{tabular}{|c|c|c|c|c|c|}
\hline No. of composition & Nomenclature & Ethanol, $\%$ & Pine resin, $\%$ & Rapeseed oil, $\%$ & Aluminum, $\%$ \\
\hline 1 & EthSin45A & 45 & 45 & - & 10 \\
\hline 2 & Ethanol & 100 & - & - & - \\
\hline 3 & EthSin45O & 45 & 45 & 10 & - \\
\hline 4 & EthSin50 & 50 & 50 & - & - \\
\hline 5 & EthSin25 & 25 & 75 & - & - \\
\hline
\end{tabular}

The fuel components have the following main characteristics. For ethanol: boiling temperature $351 \mathrm{~K}$, flash temperature $285 \mathrm{~K}$, ignition temperature $296 \mathrm{~K}$, self-ignition temperature $699 \mathrm{~K}$, heat of combustion $26.78 \mathrm{MJ} / \mathrm{kg}$, combustion rate about $60 \mathrm{~kg} /\left(\mathrm{m}^{2} \cdot\right.$ hour $)$. For rapeseed oil: boiling temperature $477 \mathrm{~K}$, flash temperature $513 \mathrm{~K}$, ignition temperature $598 \mathrm{~K}$. For pine resin: boiling temperature $523 \mathrm{~K}$, flash temperature: $439 \mathrm{~K}$, ignition temperature $453 \mathrm{~K}$, self-ignition temperature $676 \mathrm{~K}$. The size of aluminum particles was 50-250 $\mu \mathrm{m}$.

\section{Experimental Setup and Methods}

High-temperature gas medium was generated in a hollow ceramic tube of a muffle furnace. The inside diameter of the ceramic tube is $40 \mathrm{~mm}$, its length is $450 \mathrm{~mm}$; the temperature was controlled in the ranges from 300 to $1500 \mathrm{~K}$ using an in-built type $S$ thermocouple. In each series of experiments under identical initial conditions, the air in the ceramic tube of the furnace was heated up to the given temperature $T_{g}$. After it stabilized, a fuel droplet was introduced into the ceramic tube along its symmetry axis. A positioning mechanism (minirobotic arm) moved the ceramic holder with a fuel droplet on it. The positioning mechanism stopped when the droplet reached the middle of the ceramic tube.

The processes triggered by fuel droplet heating were registered by a high-speed color camera Phantom v411. Its specifications are as follows: $4200 \mathrm{fps}$ at full resolution of $1280 \times 800$ pixels; $20 \mu \mathrm{m}$ pixel size; 12 -bit depth; $16 \mathrm{~Gb}$ memory; $1 \mu \mathrm{s}$ minimum exposure; image-based auto-trigger. Video recordings of the experiments were analyzed using the Tema Automotive software. We used a software and hardware system to automatically control the initial droplet size $\left(D_{d}\right)$ and to calculate the ignition delay time $\left(t_{d}\right)$. The typical size (diameter) of droplets was about $2 \mathrm{~mm}$. The systematic error of $D_{d}$ determination with the respective video recording resolution settings of the high-speed camera did not exceed $4 \%$. The systematic error of $t_{d}$ calculation did not exceed 3\%. Random errors for sets of 5 experiments under identical conditions did not exceed $10 \%$.

\section{Results and Discussion}

The experimental study helped us determine the minimum air temperatures $T_{g}$ (Fig. 1a), at which ignite the droplets of the initial liquid fuel and different compositions of gel fuel on its basis. The minimum temperatures range from 873 to $943 \mathrm{~K}$ and go up in the following order for the fuel compositions: No. 1, No. 2, No. 3, No. 4, No. 5. Shorter ignition delay times $t_{d}$ and lower $T_{g}$ are typical of gel fuel composition No. 1 with fine aluminum particles. Droplets of pure ethanol (composition No. 2) are also ignited at $T_{g}=873 \mathrm{~K}$ but the delay is up to twice as long as that of composition No. 1. The result obtained has the following reasons. In our experiments, adding metal particles to a gel fuel intensifies the droplet heating and boosts the flammable liquid evaporation rate, since the thermal conductivity coefficient of aluminum is $422 \mathrm{~W} /(\mathrm{m} \cdot \mathrm{K})$ at $873 \mathrm{~K}$ and the thermal conductivity coefficients of ethanol and pine resin are $0.090 \mathrm{~W} /(\mathrm{m} \cdot \mathrm{K})$ and $0.128 \mathrm{~W} /(\mathrm{m} \cdot \mathrm{K})$, respectively. At high temperatures, heated metal particles at the near-surface layer of a droplet may provide multiple nucleation sites, thus increasing the evaporation rate. Metal particles may also improve the diffusion heat exchange by altering the thermophysical properties of fuel such as increasing the thermal conductivity and reducing the surface tension. Adding a gellant to liquid fuel and increasing its concentration leads to an increase in the gel fuel ignition delay (curves No. 3-5 in Fig. 1a). In such conditions, lower $T_{g}$ and $t_{d}$ are typical of composition No. 3 with rapeseed oil. This oil is the reason for the droplet dispersion when heated, which has been observed in the experiments and shown to intensify the ignition of the initial fuel droplet (or rather its small parts-dispersion products). 
Maximum differences in the ignition delay times are typical of the ambient temperatures close to the minimum $T_{g}$ required for fuel ignition. At $T_{g}>1048 \mathrm{~K}$, the ignition delay times for various compositions do not exceed $0.1 \mathrm{~s}$. In such conditions, the difference of the $t_{d}$ values by several percent is negligible and comparable to the error of a measuring instrument. Thus, when gel fuel droplets of different compositions based on the same combustible liquid are heated rapidly, neither the component composition, nor the concentration of gellant and other flammable components affect the main parameter of the process-ignition delay time.

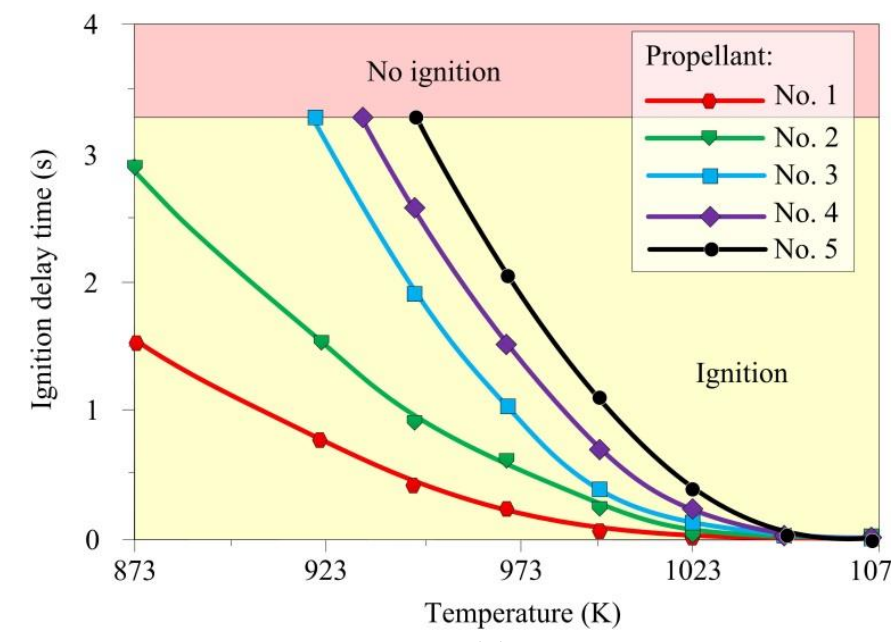

(a)
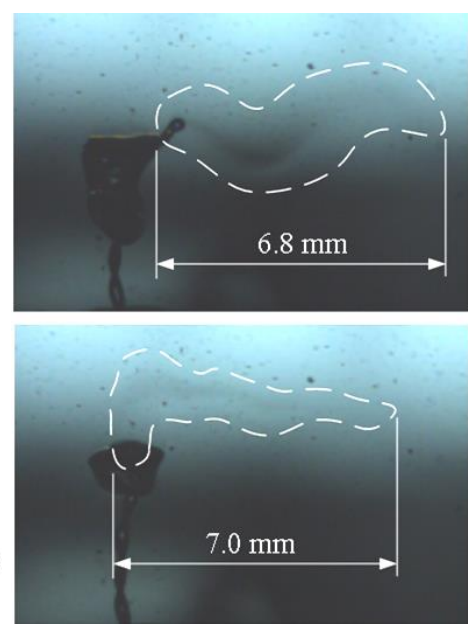

(b)
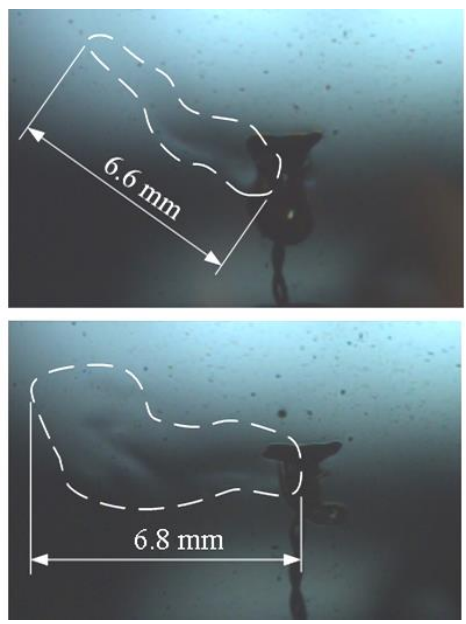

b)

Fig. 1: Ignition delay times of fuel droplets $R_{d} \approx 1.25 \mathrm{~mm}(a)$ and video frames of jetting events when heating gel fuel droplets (composition No. 4) at $T_{g}=975 \mathrm{~K}(b)$.

Fig. $1 b$ presents the video frames of jetting events during gelled droplet heating (composition No. 4 ) at $T_{g}=975 \mathrm{~K}$. Dashed lines illustrate the dimensions and shape of the zones of flammable liquid vapor jetting into the oxidizing medium. For the first time, by combining the shadow method and high-speed video recording, we recorded the jetting events and then analyzed them using the Tema Automotive software. Within the induction period (up until the fuel ignition) 2-4 jetting events were observed for compositions No. 3 - No. 5 during droplet heating. The statistical analysis of our experimental findings shows that the average speed of vapor jetting into the oxidizing medium is about $3 \mathrm{~m} / \mathrm{s}$. The size of the zones, in which the vapors slow down to zero, ranges from 6 to $8 \mathrm{~mm}$ with the initial droplet diameter of about $2 \mathrm{~mm}$.

The result obtained plays an important part for developing physical and mathematical models simulating the ignition of gel fuel droplets (Fig. 2).

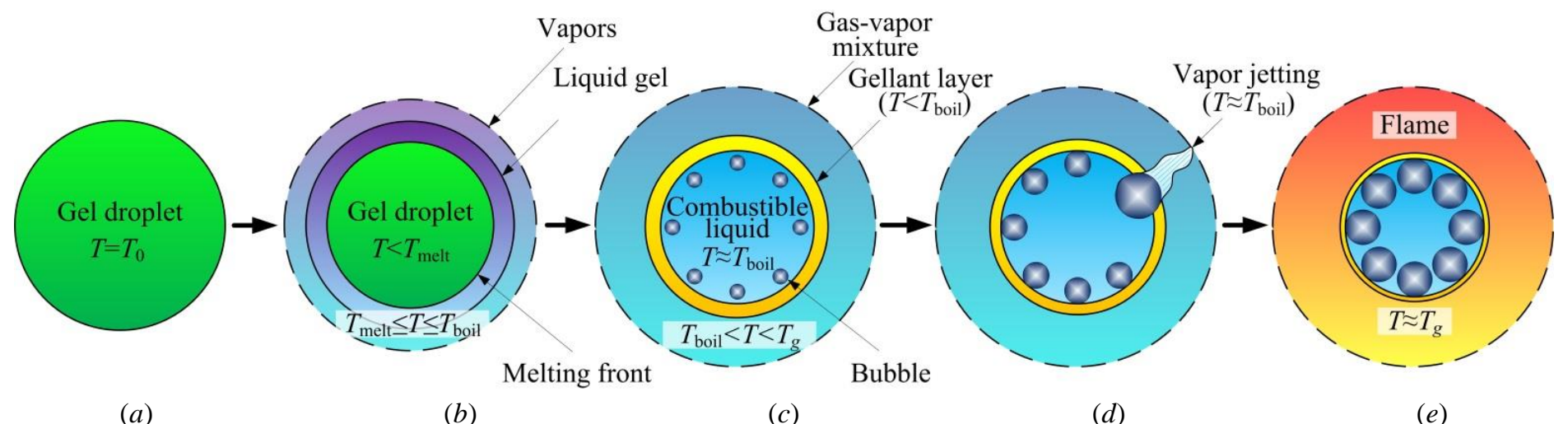

Fig. 2: Scheme of the physical model simulating the ignition of a gel fuel droplet. 
During the first period of droplet heating (Fig. 2a), two phase transformations-melting and evaporation-occur consecutively in the high-temperature oxidizing medium. The melting front is formed on the droplet surface (Fig. $2 b$ ) moves to the deep layers as the droplet heats. At the initial melting stage, the flammable liquid vapors enter the oxidizing medium from the free droplet surface. Than as a result of melting, the easily boiling component and the non-boiling component separate from each other (Fig. 2c). A layer of molten gellant is formed on the droplet surface with the liquid beneath it. Differences between the boiling temperatures of the components cause microexplosions and jetting events (Fig. 2d). As a result, combustible vapors are blown towards the droplet. The concentration of fuel increases in the forming gas-vapor mixture and the combustion becomes more vigorous (Fig. 2e).

\section{Conclusion}

For the gel fuel compositions under study, we determined the minimum ambient temperatures of 873-943 K required for the ignition as well as the dependences of gas-phase ignition delay times versus air temperature. The ignition delay times range from 0.1 to $3.3 \mathrm{~s}$. If the ignition does not start within this period, it will not occur after a longer heating time. For different gel fuel compositions based on a combustible liquid and for a combustible liquid itself, there is a maximum heated air temperature. If this temperature is exceeded, the difference of ignition delay times becomes negligible. When gel fuel droplets of different compositions based on ethyl alcohol are heated rapidly in the air heated to over $1048 \mathrm{~K}$, neither the component composition, nor the concentration of gellant and other flammable components affect the main parameter of the process-ignition delay time. Under the near-threshold fuel ignition conditions, its component composition dominates the main characteristics of the process.

This experimental study was the first to determine the characteristics of vapor jetting triggered by microexplosions during the induction period, i.e., before the gas-phase ignition of a gel fuel droplet. The speed of vapor jetting into the oxidizing medium near the droplet surface is about $3 \mathrm{~m} / \mathrm{s}$ and the size of vapor distribution zones ranges from 6 to $8 \mathrm{~mm}$. Within the induction period (up until the fuel ignition) 2-4 jetting events were observed during gelled droplet heating. However, we can conclude that gellant concentration, just as the ambient temperature, and the jet speed (or the quantity of jetting events) had a direct relationship.

Based on these experimental results, a physical model of the process was formulated, which may serve as the foundation for a mathematical model simulating the ignition of a gel fuel droplet and help refine the current mathematical models simulating the combustion of such fuels.

\section{Acknowledgements}

This study was supported by the Russian Science Foundation (project No. 18-13-00031).

\section{References}

[1] B. Natan and S. Rahimi, "The status of gel propellants in year 2000," in Combustion of Energetic Materials, K. K. Kuo and L. T. DeLuca, Eds. New York: Begell House, 2002, pp. 172-194.

[2] S. Feng, B. Hea, H. He, L. Su, Z. Hou, W. Nie, X. Guo, "Experimental studies the burning process of gelled unsymmetrical dimethylhydrazine droplets under oxidant convective conditions," Fuel, vol. 111, pp. 367-373, 2013.

[3] D. P. Mishra, A. Patyal, M. Padhwal, "Effects of gellant concentration on the burning and flame structure of organic gel propellant droplets," Fuel, vol. 90, pp. 1805-1810, 2011.

[4] Y. C. Kevin, T. L. Pourpoint, S. F. Son, R. P. Lucht, "Microexplosion investigation of monomethylhydrazine gelled droplet with OH planar laser-induced fluorescence," Journal of Propulsion and Power, vol. 29, pp. 1303-1310, 2013.

[5] F. A. Williams, Combustion Theory, Boulder (United States): Westview Press, 1985.

[6] Y. B. Zeldovich, G. I. Barenblatt, V. B. Librovich, G. M. Makhviladze, Mathematical Theory of Combustion and Explosions. New York: Plenum, 1985.

[7] V. N. Vilyunov and V. E. Zarko, Ignition of Solids. Amsterdam (Nederland): Elsevier Science Publishers, 1989.

[8] S. Okajima and S. Kumagai, "Further investigation of combustion of free droplets in a freely falling chamber including moving droplets," in Proceedings of the Fifteenth Combustion Symposium, 1998, pp. 401-407. 